\title{
About When We Collide: A Generative and Collaborative Sound Installation
}

\author{
PerMagnus Lindborg ${ }^{*}$, Joyce Beetuan Koh ${ }^{\dagger}$ \\ *School of Art, Design, and Media, Nanyang Technological University \\ ${ }^{\dagger}$ Nanyang Academy of the Fine Arts, Singapore \\ permagnus@ntu.edu.sg
}

\begin{abstract}
The idea for When We Collide sprang from Douglas Hofstader's metaphor of creativity as the meeting between records and record players, appearing in his 1979 book "Gödel, Escher, Bach: An Eternal Golden Braid". In our case, the records are soundfiles, whilst the record player is a generative system. The player analyses, selects, mixes, transforms, and spatialises the material created by the composers (monophonic and quadraphonic soundfiles). The system negotiates between algorithms that tend towards monotony (in terms of loudness, spatialisation, and frequency spectrum) and algorithms that tend towards variability (in terms of soundfiles, transformations, and scenes). In a nutshell, the installation is a space where sonic ideas collide and co-exist.
\end{abstract}

Keywords: generative, installation, composition

\section{Introduction}

When We Collide is a generative sound installation based on audio material contributed by six composers. It is a development from two previous productions: On the String, inspired by String Theory, commissioned by the Singapore Arts Festival 2010; and The Canopy, presented at International Computer Music Conference 2011 and World Stage Design Festival 2013. These works are about our fascination with the building blocks of the universe, in particular, the distinctive decay signatures of elementary particles. Imagine attributing their distinctive features to sonic characteristics. What musical possibilities arise from the idea of particle collisions, interactions, and decay?

Originally, When We Collide was conceived as an interactive installation of four independent large physical structures of strings, configured in the Chinese character 'ru' / $入$ which means 'to enter' (Figure 1). The visitor, in the role of a dancer-musician, would modify the sonic characteristics of a particle by movements. The image evoked is a pas de deux with

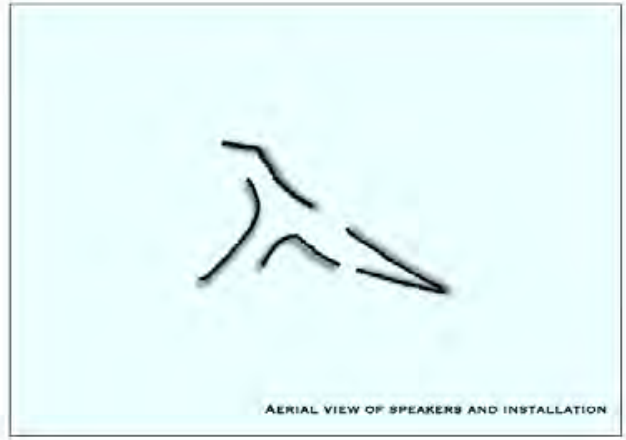

Figure 1. Installation draft (top-down view) of string structures resembling a Chinese character.

an invisible, dark super partner - dancing elementary particles - yielding a multimodal experience that mediates between the eyes, ears and body. The construction of this scale became too resource intensive. Many discussions ensued and various guises of the installation were deliberated. One of the guises was inspired by lannis Xenakis' drawings of the Montreal Exposition 1967 (Figure 2). 


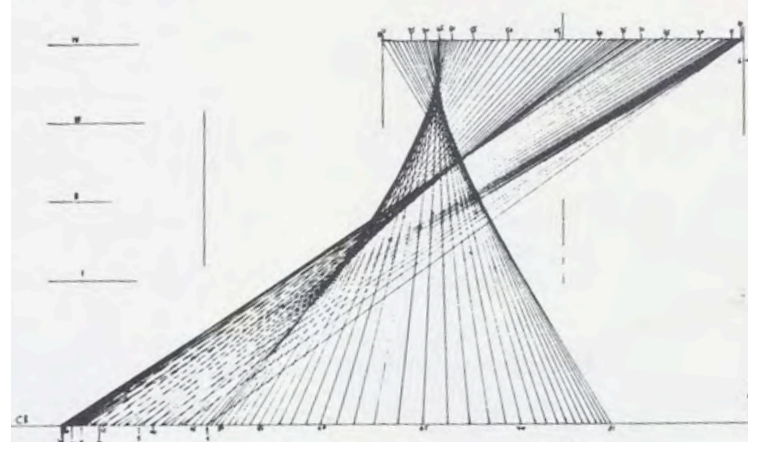

Figure 2. A visualisation of the pitch and architectural structures by lannis Xenakis.

Our structure was to have strings fixed with stretch sensors. The intention was to 'wrap the space' in a specific pattern reminiscent of the particles decay signatures (Figure 3 ).

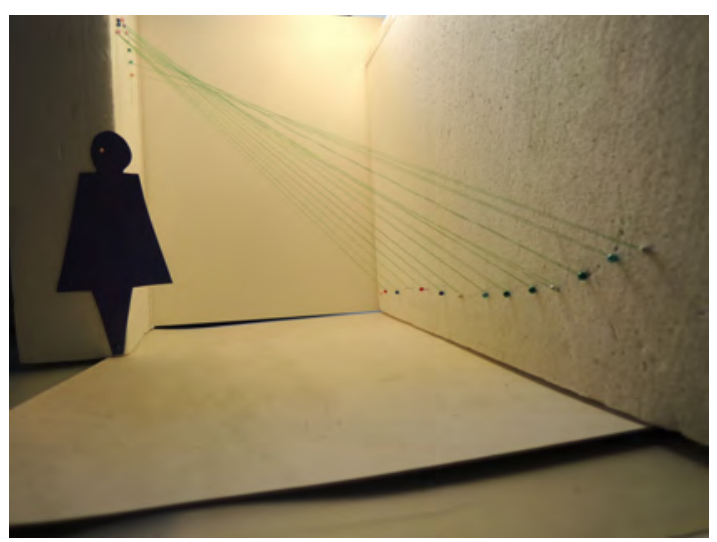

Figure 3. Mockup of the staging for On The String. Note the strings across the space.

\section{A collaborative and generative instal- lation}

In When We Collide, we have revisited the concept of particle-collisions. Instead of simulating the concept with physical material, we could lean on our aural and spatial senses. What could be more apt than to have six composers, each has a distinct language, alluding to the decay signatures of particles, compose musical material for a generative system that is designed to allow collision, morphing and becoming. The invited composers were Andrián Pertout, Seongah Shin, Stefano Fasciani, Dirk Stromberg. The authors also contributed material. There were thus six artists in all, each of us providing five quadraphonic soundfiles and twenty monophonic soundfiles: quadraphonic files are 55 seconds long, and mono files 5 seconds.

\section{Player design}

The ideas behind the 'player' come from more than one source of inspiration; important to mention are John Cage's Williams Mix, the Freq-out installations curated by $\mathrm{CM}$ von Hauswolff (where one of us, Lindborg, participated), and our own previous work, in particular Lindborg's Khreia for orchestra (2002) and Koh's FingerPrints (2014).

First, we imagined the player simply as a jukebox - "put another nickel in, then you'll hear the music spin" - that would pick at random among the 30 quadraphonic files that the artists had composed. The selection mechanism developed into a small set of rules where the probability vector for the quadraphonic files is updated in cascade depending on which files have been played earlier. Also, the presently playing quad files limit which monophonic files can be selected, as well as when, and where (in the surround panning) they can appear (Figure 4).

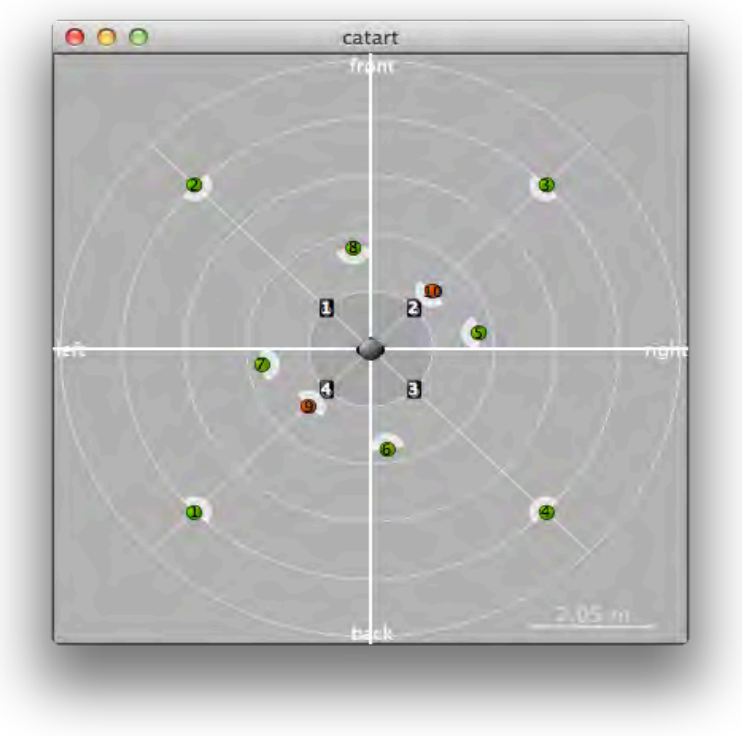

Figure 4. The surround panning space as it appears in the IRCAM spat user interface, in a 'bird's eye' perspective. The filled circles represent sound sources, and the squares outputs e.g. physical loudspeakers, or virtual sources for binaural reproduction. 


\section{Instruments}

We defined four virtual instruments (or 'subplayers'), striving to integrate their functioning and sonic output, so as to yield the impression of an ensemble.

The first instrument, 'quadbase', consists of two quad sub-players taking turns, which may sometimes overlap by a certain amount. Their continuous output is analysed for loudness (using Ircamdescriptors) and main directivity, and a self-correcting mechanism tries to adjust the spatialisation to make the overall output as even as possible.

The second and third instruments, nicknamed 'recomposer' and 'cloud', are both based on CataRT (Diemo Schwarz 2015). They try, in different ways, to imitate what the quad players are doing. One selects among the 120 mono files originally submitted by the composers, and the other chops these files up into approximately 3000 fragments in order to produce a cloud-like sound (Figure 5). Both receive a mono mix and file IDs from the quadplayers in order to determine the output.

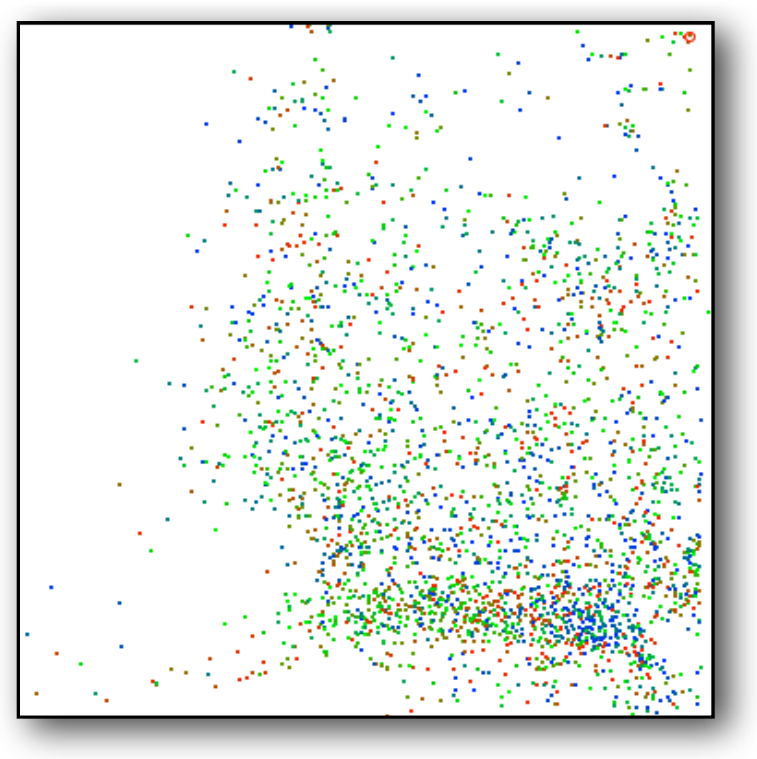

Figure 5. Approximately 3000 dots visualised in the CataRT LCD interface (Schwarz 2015). They represent sonic descriptors for short fragments of the mono files, projected onto a plane with spectral flatness and periodicity on $x$ and $y$ axes, respectively.
The fourth instrument, consisting of two sub-players so it might be called a duo, is called 'mirror solos'. It merges mono files two by two in cross-synthesis (using the supervp object from IRCAM). While the first two players (quad and CataRT-quad) respect the composers' original work, the last two (CataRTcloud and the crossynths) produce new and quite unpredictable output.

\section{Overall control}

Finally (this was the last phase in the design work), an overall mechanism (at the middle level in the player structure; macro - meso micro) decides which player plays when, by applying fade-ins and fade-outs according to a cyclical process (based on Lindborg's Graviton Dance pieces and our collaboration On The String, mentioned earlier), and 'freezes', that are applied stochastically. Figure 6 illustrates the information flow in the player system.

The overall output was intended to have the character of 'chill-out electroacoustic music', yet the material and the players colluded to make it rather active at some moments. The superposition of deterministic and probabilistic mechanisms created a player structure where the interactive design and composed material collide.

\section{Acknowledgement}

The production of When We Collide was enabled by a grant from National Arts Council of Singapore. The first presentation took place at Soundislands Festival, 19-23 August 2015.

\section{References}

Hauswolff $M$ von, Lindborg PM, Grönlund T., Harding M., Kirkegaard, J., LaBelle B., Nilsen B.J., Nisunen, P., Petursson, F., Pomassl, F., Tankred, K., Thirwell, J., Urstad, M. \& Winderen, J. (2003, 2004, 2005, 2006, 2008, 2012, 2013, 2014). Freq-out \#. Series of site-specific collaborative sound installations. Each instance is specifically created for the acoustics and context of the location. URL http://www.freq-out.org.

Koh JBT, Lindborg, PM, Stromberg D, Chian $\mathrm{KH}$, \& Lim WW. (2010) On the String, theatre of 
music. Multimedia performance (6o min.) Commissioned by National Arts Council for Singapore Arts Festival.

Lindborg PM \& Koh, Joyce Beetuan (2011, August). "Multidimensional spatial sound design for 'On the String'". Proceedings of the International Conference of Computer Music (ICMC), University of Huddersfield, UK. July 2011.
Lindborg PM (2013). Graviton Dance - Semantic. Electroacoustic music composition with generative $3^{D}$ audio diffusion; concert (8 min.) \& interactive installation versions. International Computer Music Conference 2013. Perth, Australia. 11-17 August 2013.

Schwarz D, Beller G, Verbrugghe B \& Britton S (2006). Real-time corpus-based concatenative synthesis with CataRT. Proceedings of DAF-X, p. 279282, Montreal 2006.

When We Collide, player overview

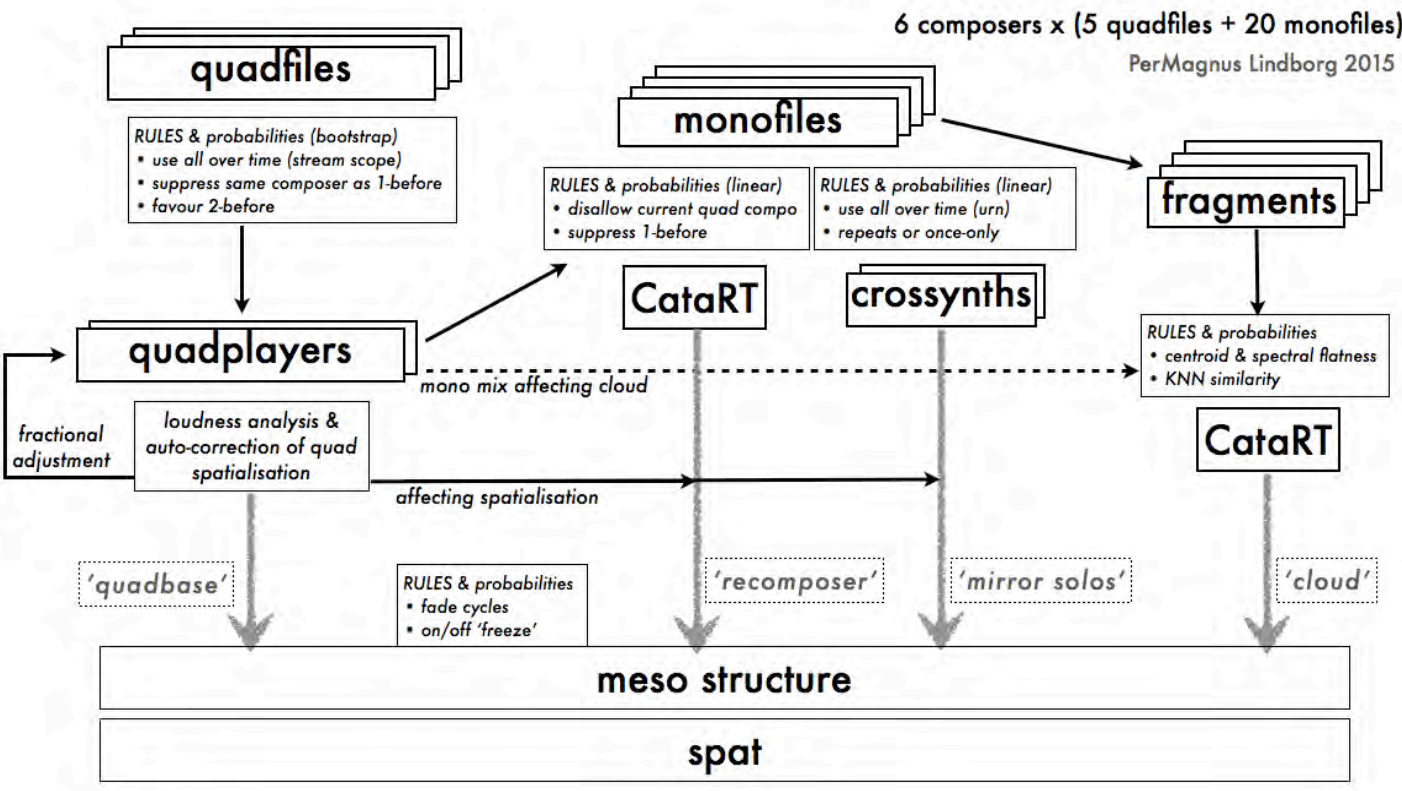

Figure 6. Overview of player design. Top: boxes represent sound files (provided by the artists), and descriptions of selection algorithms. Middle: boxes represent instruments, producing in all 18 channels of audio. The arrows indicate some of the ways that sub-systems influence each other. Bottom: boxes represent meso structure (i.e. control layer) and spatialisation. 